\title{
A SYMMETRIC STAR BODY THAT TILES BUT NOT AS A LATTICE
}

\author{
SHERMAN K. STEIN
}

\begin{abstract}
A classical question in convex bodies runs as follows: "If translates of a fixed convex body $K$ in Euclidean space can be packed with a certain density, is it possible to find a lattice packing by translates of $K$ that is at least as dense?" This suggests a similar question for star bodies, which is answered negatively in the present paper. It is shown that there is a centrally-symmetric star body that tiles ten-dimensional Euclidean space but does not tile it in a lattice manner.
\end{abstract}

We shall construct a star body that tiles Euclidean space $R^{10}$ but not as a lattice. This body consists essentially of 81 unit cubes arranged in the form of a cross that has a central cube from which emanates, at each of its twenty nine-dimensional facets, an arm composed of four cubes. This body is symmetric with respect to the center of its central cube and with respect to the interchange of axes.

1. Preliminaries. Let $R^{n}$ denote $n$-dimensional Euclidean space, consisting of the points $\left(x_{1}, \cdots, x_{n}\right), x_{i} \in R^{1}$. A set $K \subseteq R^{n}$, homeomorphic to the $n$-cell, we shall call a star body if there is a point in $K$ from which all of $K$ is visible; more precisely, if there is a point $A \in K$ such that for every point $B \in K$ the chord $A B$ lies in $K$. In particular any convex body is a star body.

Let $V=\left\{v_{1}, v_{2}, \cdots, v_{n}, \cdots\right\}$, where $v_{1}=(0,0, \cdots, 0)$, be a set of vectors in $R^{n}$ with the property that for $i \neq j$ the interior of $v_{i}+K$ is disjoint from the interior of $v_{j}+K$. The family $v_{1}+K, v_{2}+K, \cdots$ is called a packing of $R^{n}$ by translates of $K$. If, furthermore, $R^{n}=\bigcup_{i}\left(v_{i}+K\right)$, then the packing is called a tiling of $R^{n}$. If the set $V$ forms a lattice (a subgroup of $R^{n}$ without limit points) then one speaks of a lattice packing or lattice tiling by translates of $K$. This topic is surveyed by C. A. Rogers in [2, pp. 1-20] in case $K$ is a convex body. No example is known of a convex body whose translates can be packed more densely then they can be as a lattice.

Presented to the Society, May 7, 1971 under the title $A$ nonlattice tiling by a star body; received by the editors October 19, 1971.

AMS (MOS) subject classifications (1970). Primary 52A45; Secondary 10E30.

Key words and phrases. Packing, tiling, convex body, star body, lattices, nonconvex body, abelian group.

(C) American Mathematical Society 1973 
The assertion that, for $i \neq j$, the interiors of $v_{i}+K$ and $v_{j}+K$ are disjoint is equivalent to the assertion that $v_{j}$ is the only element of $V$ in the interior of $v_{j}+[(-K)+K]$, where $-K=\{-x \mid x \in K\}$ and

$$
(-K)+K=\{x+y \mid x \in-K, y \in K\} \text {. }
$$

This suggests the question of determining, for a set $K^{\prime} \subset R^{n}$, how dense a lattice $V=\left\{v_{1}, v_{2}, \cdots\right\}$ exists such that for each $j, v_{j}$ is the only member of $v_{1}, v_{2}, \cdots$ in the interior of $v_{j}+K^{\prime}$. Since not every star body $K^{\prime}$ is of the form $(-K)+K$ for some set $K$, the first problem, concerning tiling, even for star bodies is more general than the second. Zassenhaus in [6] surveys results on the second problem. Wolff in [4] constructs a star body $K^{\prime}$ in $R^{2}$, symmetric with respect to the origin, such that lattices do not provide the densest set $V$ for the second problem.

We shall construct a star body $K$ in $R^{10}$, symmetric with respect to the origin, such that translates of $K$ tile $R^{10}$ but there is no lattice tiling of $R^{10}$ by translates of $K$. This body is a special instance of a $(k, n)$-cross with notches. A $(k, n)$-cross, introduced in [3], is defined as follows. For any positive integer $k$ and Euclidean space $R^{n}$, a $(k, n)$-cross consists of $2 k n+1$ parallel unit cubes, one central cube together with an arm of length $k$ stuck onto each of the $2 n$ facets of the central cube. More precisely, it is a translate of the set of unit cubes parallel to the axes and whose centers are at

$$
(0,0, \cdots, 0),( \pm i, 0, \cdots, 0),(0, \pm i, 0, \cdots, 0), \cdots,(0,0, \cdots, \pm i)
$$

$i=1,2, \cdots, k$. Observe that a $(k, n)$-cross is centrally symmetric.

It is trivial to check that a $(k, 1)$-cross (i.e., an interval of length $1+2 k$ ) tiles $R^{1}$, and only as a lattice. Similarly, in $R^{2}$, it is easy to see that the $(1,2)$-cross tiles, but only as a lattice; no other $(k, 2)$-cross tiles $R^{2}$. In $R^{3}$ both the $(1,3)$-cross and the $(2,3)$-cross tile, but only as a lattice. No other $(k, 3)$-crosses tile $R^{3}$. However, as we have mentioned, the $(4,10)$-cross tiles $R^{10}$ but not as a lattice. Whether ten is the smallest dimension at which this phenomenon occurs is not known. The proof rests on two lemmas.

2. Two lemmas. Let $S$ be a set of $q$ elements and $S^{n}=S \times S \times \cdots \times S$ be the Cartesian product of $n$ copies of $S, n \geqq 3$. A subset $M$ of $S^{n}$ is called a perfect cover of $S^{n}$ if each element of $S^{n}$ differs from exactly one member of $M$ in at most one coordinate. (A set of chess "rooks" placed at the elements of $M$ attack each element in $S^{n}$ exactly once.)

LEMMA 1 (ZAREMBA [5]). Let $q$ be a power of a prime, $r$ an integer $\geqq 2$, and $n=\left(q^{r}-1\right) /(q-1)$. Then there is a perfect cover of $S^{n}$, and it consists of $q^{n-r}$ elements. 
For a shorter proof see [1].

Let $G$ be a group and $A$ and $B$ be subsets of $G$. If each element of $G$ is uniquely expressible in the form $a b, a \in A, b \in B, G$ is called the product of $A$ and $B$. $G$ is also said to be factored by the subsets $A$ and $B$.

The next lemma enables us to lift a factoring from a group $G^{*}$ to any group $G$ of which $G^{*}$ is a homomorphic image.

Lemma 2. Let the group $G^{*}$ be the product of subsets $A^{*}$ and $B^{*}$. Let $G$ be another group and $f: G \rightarrow G^{*}$ be a homomorphism from $G$ onto $G^{*}$. Let $A$ be any subset of $G$ such that the restriction of $f$ to $A$ is a bijection between $A$ and $A^{*}$. Let $B=f^{-1}\left(B^{*}\right)$. Then $G$ is the product of $A$ and $B$.

Proof. We first show that any element in $G$ is of the form $a b$. For $x \in G$ consider $f(x)=a^{*} b^{*}, a^{*} \in A^{*}$ and $b^{*} \in B^{*}$. Select $a \in A$ such that $f(a)=a^{*}$. Then $f\left(a^{-1} x\right)=\left(a^{*}\right)^{-1} a^{*} b^{*}=b^{*}$. Hence $a^{-1} x$ is an element $b$ in $B$. Thus $x=a b$.

We next establish uniqueness. Let $a_{1} b_{1}=a_{2} b_{2}$, where $a_{1}, a_{2}$ are in $A$ and $b_{1}, b_{2}$ are in $B$. Then

$$
f\left(a_{1} b_{1}\right)=f\left(a_{2} b_{2}\right) \text { or } f\left(a_{1}\right) f\left(b_{1}\right)=f\left(a_{2}\right) f\left(b_{2}\right) .
$$

Since $G^{*}$ is the product of $A^{*}$ and $B^{*}, f\left(a_{1}\right)=f\left(a_{2}\right)$, and therefore $a_{1}=a_{2}$. Cancellation yields $b_{1}=b_{2}$. This proves Lemma 2 .

3. Proof of the theorem. The two lemmas, combined with the following observations, easily yield a proof of the theorem. Consider a tiling of $R^{n}$ by crosses whose arms are parallel to the axes. It is not clear whether, for each $i, 1 \leqq i \leqq n$, the $i$ th coordinate of any two crosses in the tiling differ by an integer. In order to be sure that the $i$ th coordinates do differ by an integer we notch the crosses in the manner indicated in the following diagram. This notching can be done in such a way that the notched cross is a centrally symmetric star body; as notches use a shallow cap of a sphere.

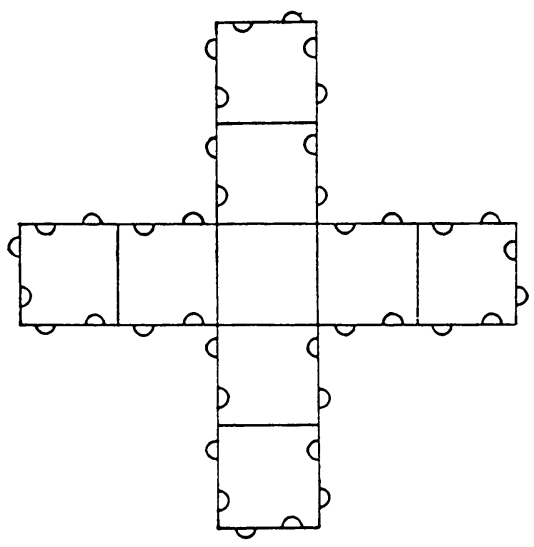


Place the centers of the notch and dent on the facets perpendicular to the $x_{i}$-axis in such a way that they determine a line parallel to the $x_{i+1}$-axis, $i=1,2, \cdots, n$, where $x_{n+1}$ is interpreted to be $x_{1}$.

THEOREM. The notched $(4,10)$-cross tiles $R^{10}$, but not as a lattice.

Proof. Lemma 1 with $q=3^{2}$ and $n=\left(q^{2}-1\right) /(q-1)=q+1=3^{2}+1=10$ shows that for a set $S$ with nine elements, the set $S^{10}$ has a perfect cover, $M$. Now let $S$ be $Z_{9}=Z /(9 Z)$, the cyclic group of order nine. For $z \in Z$ let $\bar{z}$ denote the element $z+9 Z$ of $Z_{9}$. Then $S^{10}$ forms a group, $Z_{9} \times Z_{9} \times \cdots$ $\times Z_{9}$, which we denote $G^{*}$. Let $A^{*}=\{(\overline{0}, \overline{0}, \cdots, \overline{0})\} \cup\{( \pm \bar{z}, \overline{0}, \overline{0}, \cdots, \overline{0})\} \cup$ $\{(\overline{0}, \pm \bar{z}, \overline{0}, \cdots, \overline{0})\} \cup \cdots \cup\{(0,0, \cdots, \pm \bar{z})\}, 1 \leqq z \leqq 4 . A^{*}$ has 81 elements. Let $B^{*}=M$. Since $M$ is a perfect cover of $S^{10}, G^{*}$ is the product of $A^{*}$ and $B^{*}$.

Let $f$ be the homomorphism $f: Z^{10} \rightarrow Z_{9}^{10}$ defined by

$$
f\left(z_{1}, z_{2}, \cdots, z_{10}\right)=\left(\bar{z}_{1}, \bar{z}_{2}, \cdots, \bar{z}_{10}\right) \text {. }
$$

Let $A \subset Z^{10}$ be the 81 lattice points that are the centers of the cubes of the $(4,10)$-cross whose center is at the origin of $R^{10}$. Clearly $f \mid A$ is a bijection between $A$ and $A^{*}$. By Lemma $2, Z^{10}$ is the product of $A$ and $f^{-1}\left(B^{*}\right)$. Consequently, the $(4,10)$-cross tiles $R^{10}$. Hence the notched $(4,10)$-cross tiles $R^{10}$.

Next we show that the notched $(4,10)$-cross does not tile $R^{10}$ as a lattice. Assume that $R^{10}$ is tiled in a lattice manner by translates of the notched $(4,10)$-cross. The notching of the crosses forces their centers to have integer coordinates. Thus $Z^{10}$ would be the product of the set $A$ consisting of the 81 elements described above and a subgroup $H$ of $Z^{10}$. Let $h: Z^{10} \rightarrow Z^{10} / H$ be the natural homomorphism. Observe that $Z^{10} / H$ has order 81 and that $h \mid A$ is a bijection from $A$ to $Z^{10} / H$. Let $g_{i} \in Z^{10} / H, i=1,2, \cdots$, 10 , be $h\left(e_{i}\right)$, where $e_{i}$ is a basic unit vector in $Z^{10},(0,0, \cdots, 1, \cdots, 0)$, a 1 in the $i$ th place. Thus the 80 nonzero elements $Z^{10} / H$ are $\pm g_{i}, \pm 2 g_{i}$, $\pm 3 g_{i}, \pm 4 g_{i}, i=1,2, \cdots, 10$. Clearly, the order of each $g_{i}$ is at least 9 .

Now the group $Z^{10} / H$ is either $Z_{3} \times Z_{3} \times Z_{3} \times Z_{3}, Z_{9} \times Z_{9}, Z_{27} \times Z_{3}$, $Z_{9} \times Z_{3} \times Z_{3}$ or $Z_{81}$. The case $Z_{3} \times Z_{3} \times Z_{3} \times Z_{3}$ is ruled out since all of its elements have order less than 9 . The case $Z_{9} \times Z_{9}$ is out since each $g_{i}$ would have order 9 ; the 20 element $\pm 3 g_{i}$ would have order 3 . But there are only eight elements of order 3 in $Z_{9} \times Z_{9}$. To rule out $Z_{27} \times Z_{3}$ note that $Z_{27} \times Z_{3}$ has eight elements of order 3 . Thus, four of the elements of $g_{1}, \cdots, g_{10}$, say $g_{1}, g_{2}, g_{3}, g_{4}$, have order 9 , and $\pm 3 g_{1}, \pm 3 g_{2}, \pm 3 g_{3}, \pm 3 g_{4}$ are the eight elements of order 3 . Then the 24 elements $\pm g_{i}, \pm 2 g_{i}, \pm 4 g_{i}, i=1,2,3,4$, are of order 9 . However $Z_{27} \times Z_{3}$ has only 18 elements of order 9 . To rule out $Z_{9} \times Z_{3} \times Z_{3}$, note that $Z_{9} \times Z_{3} \times Z_{3}$ has 26 elements of order 3 , but only the 20 elements, $\pm 3 g_{1}, \pm 3 g_{2}, \cdots, \pm 3 g_{10}$, may be of order 3 . The 
case $Z_{81}$ can be ruled out as follows. Considering $Z_{81}$ as the set $\{0,1,2, \cdots$, $80\}$ under addition modulo 81 , let $G_{81}=\{i \mid 1 \leqq i \leqq 80,(i, 81)=1\}$, a subset containing $q\left(3^{4}\right)=54$ elements, but not closed under addition. It is no restriction to assume that $g_{1}=1$. If

$$
Z_{81}-\{0\}=\bigcup_{i=1}^{10}\left\{ \pm g_{i}, \pm 2 g_{i}, \pm 3 g_{i}, \pm 4 g_{i}\right\}
$$

then $G_{81}$, regarded as a group under multiplication modulo 81 may be assumed to be the product of $\left\{g_{1}, g_{2}, \cdots, g_{9}\right\}$ and $\{ \pm 1, \pm 2, \pm 4\}$. It is then easy to show that the set $\left\{g_{1}, g_{2}, \cdots, g_{9}\right\}$ is, up to changes of sign, $\left\{8^{0}, 8^{1}, \cdots, 8^{8}\right\}$. But $3 \cdot 8^{0}=3 \cdot 1=3$ while $(-3) \cdot 8^{3}=(-3)(26)=3$. Thus $3 \cdot 8^{0}=(-3) \cdot 8^{3}$ and the alleged decomposition of $Z_{81}$ cannot exist.

Hence there is no lattice tiling of $R^{10}$ by notched $(4,10)$-crosses. This completes the proof of the theorem.

4. Remarks. The tiling constructed for the notched $(4,10)$-cross is what Zassenhaus in [6] calls a tiling using a "lattice with a base", that is, the union of a finite number of translates of a lattice. It is further evidence in favor of an affirmative answer to his question: "Is it reasonable to assume that lattices with a base form a pattern of optimal packings?"

A similar argument shows that the notched $(3,5)$-semicross tiles $R^{5}$ but not as a lattice. The $(3,5)$-semicross, defined in [3], consists of a unit cube with arms of length three stuck on one of each pair of opposite facets. It is made up of 16 cubes and is not centrally-symmetric.

It may be that the notches are not necessary to insure that the coordinates of centers differ by integers in the case of crosses. However, the $(1,3)$-semicross can tile $R^{3}$ in such a way that the coordinates of the centers do not differ by integers. A $(1,3)$-semicross $S$ consists of translates of the four unit cubes whose centers are at $(0,0,0),(1,0,0),(0,1,0)$ and $(0,0,1)$. Consider the set of translates $v+S$ where $v$ is of the form $(n,-n, n+2 t)$ or $(m+1,-m+1, m+2 s)$ where $m, n, s, t$ run through all integers. The union of these translates is a cylinder in the form of a wall four cubes thick with generator parallel to the $x_{3}$-axis. Copies of this wall tile $R^{3}$, and can be moved parallel to the $x_{3}$-axis independently of each other.

\section{REFERENCES}

1. G. Losey, Note on a theorem of Zaremba, J. Combinatorial Theory 6 (1969), 208-209. MR 39 \#1333.

2. C. A. Rogers, Packing and covering, Cambridge Tracts in Math. and Math. Phys., no. 54, Cambridge Univ. Press, New York, 1964. MR 30 \#2405.

3. S. K. Stein, Factoring by subsets, Pacific J. Math. 22 (1967), 523-541. MR 36 \#2517. 
4. M. R. Von Wolff, A star domain with densest admissible point set not a lattice, Acta Math. 108 (1962), 53-60. MR 26 \#2400.

5. S. K. Zaremba, Covering problems concerning Abelian groups, J. London Math. Soc. 27 (1952), 242-246. MR 13, 817.

6. $\mathrm{H}$. Zassenhaus, Modern developments in the geometry of numbers, Bull. Amer. Math. Soc. 67 (1961), 427-439. MR 24 \#A1887.

Department of Mathematics, University of California, Davis, California 95616 